\title{
Dinámica del crecimiento del bosque húmedo tropical, 19 años después de la cosecha bajo cuatro sistemas de aprovechamiento forestal en la Península de Osa, Costa Rica
}

\author{
Growth dynamics of tropical rainforest, \\ 19 years after harvest under \\ four forest harvesting systems in the \\ Osa Peninsula, Costa Rica
}

\author{
Ruperto Quesada Monge \\ Luis Acosta Vargas ${ }^{2}$ \\ Michael Garro Chavarría ${ }^{3}$ \\ Marvin Castillo Ugalde ${ }^{4}$
}

Fecha de recepción: 20 de enero del 2012

Fecha de aprobación: 19 de marzo del 2012 


\section{Resumen}

Dentro del proyecto "Monitoreo de ecosistemas forestales para el fortalecimiento de estrategias de conservación y uso de bosques: una contribución a la iniciativa Costa Rica Carbono Neutral.", se llevo a cabo el estudio de la dinámica del crecimiento del bosque húmedo tropical, diecinueve años después de la cosecha bajo cuatro sistemas de aprovechamiento forestal.

Por medio de la medición de todos los árboles ( $d \geq$ $10 \mathrm{~cm}$ ), se identificaron botánicamente los ingresos en tres sitios de la Península de Osa (Estero Guerra, Dos Brazos de Río Rincón y Los Mogos), donde se instaló cuatro parcelas permanentes de muestreo (PPM) de I ha, establecidas en el año 1990.

En ese momento $y$, posteriormente, quince $y$ diecinueve años (2007 y 201 I, respectivamente), se analizó el estado del bosque primario después de aplicar cuatro sistemas de aprovechamiento forestal: Sistema de aprovechamiento con bueyes (SACB), Sistema de aprovechamiento mejorado (SATM), Sistema de aprovechamiento tradicional (SATT) y Sistema de aprovechamiento bueyestractor, (SABT).

El análisis de la dinámica del bosque se realizó con base en el crecimiento, tasas de mortalidad, de reclutamiento y de recambio. El incremento corriente anual (ICA) promedio para el periodo evaluado varió de los 2,47 a los 3,56 mm/ año. El análisis estadístico mostró que el valor ICA presenta una distribución normal y homocedasticidad, y el análisis de varianza indica que no existen diferencias significativas entre los ICA de cada sistema de aprovechamiento ( $p=$ $0,3410)$.

La tasa de mortalidad promedio para los cuatro sistemas de aprovechamiento es de 1,301\% y la de reclutamiento de 1,869\%, mientras que la tasa de recambio anual es de 0,569\%. El análisis estadístico comprobó que las tasas de mortalidad, reclutamiento y recambio presentan una distribución normal y homocedasticidad, y, por su parte, el análisis de varianza mostró que las diferencias entre los aprovechamientos no son significativas (mortalidad: $p=0,24$ I 2; reclutamiento: $p=0,0675$; recambio: $p=0,1$ | 59). Las pruebas $t$ apareadas mostraron que las tasas de mortalidad y reclutamiento son estadísticamente iguales dentro de cada aprovechamiento (SACB: $p=0,4992$; SATM: $p=0,9238$; SATT: $p=0,9080 ;$ SABT: $p=0,8065$ ), lo que confirma el equilibrio dinámico en que se encuentran los bosques intervenidos en estudio.

\section{Palabras clave}

Aprovechamiento forestal, dinámica, crecimiento, mortalidad y reclutamiento.

\section{Abstract}

Within the project "Monitoring of forest ecosystems to strengthen conservation strategies and forest use: a contribution to Costa Rica carbon neutral initiative", the dynamic growth of the tropical rainforest was studied, nineteen years after harvest under four systems of forest use. Through the measurement of all trees $(\mathrm{d} \geq 10 \mathrm{~cm})$, three revenue sites were botanically identified in the Peninsula de Osa (Estero Guerra, Dos Brazos de Río Rincón and Los Mogos). Four permanent sampling parcels (PPM) of I HA were installed in 1990.

At that time and subsequently, fifteen and nineteen years later (2007 and 2011, respectively), the status of the primary forest was examined after applying four logging systems: oxen harvesting system (SACB), improved harvesting system (SATM), traditional harvesting system (SATT) and oxen-tractor system (SABT).

The analysis of the forest dynamic was made based on growth, mortality, recruitment and replacement rate. The current annual increment (ICA) average for the period under review ranged from 2,47 to 3,56 mm / year. Statistical analysis showed that the ICA value has a normal distribution and homoscedasticity and the analysis of variance indicated no significant differences between the ICA of each logging system $(p=0,34 \mid 0)$.

The average mortality rate of the four logging systems is 1,301\%, the recruitment rate is 1,869\%. The annual turnover rate is $0,569 \%$. Statistical analysis found that mortality; recruitment and replacement rates show a normal distribution and homoscedasticity. On the other hand, analysis of variance showed that differences between the different logging systems are not significant (mortality: $P=0,24$ I2; recruitment: $p=0,0675$; parts: $p=0,1$ I 59). Paired tests showed that the mortality and recruitment rates are statistically the same within each logging (SACB: $p=0,4992 ;$ SATM: $p=0,9238 ;$ SATT: $p=0,9080 ;$ SABT: $p=0,8065)$, confirming the dynamic equilibrium of the forests under study.

\section{Key words}

Forest planning, dynamic, growth, mortality and recruitment. 


\section{Introducción}

\section{Antecedentes del manejo forestal en Costa Rica}

La primera Ley Forestal data de 1969 y su implementación fue muy limitada debido, entre otras cosas, a la poca disponibilidad de recursos económicos y de personal técnico. La primera escuela forestal a nivel universitario inició sus labores en 1975 y los primeros ingenieros forestales se graduaron en 1978 (Food and Agriculture Organization of the United Nations [FAO], 1995).

A principios de la década de los 80 se decretó necesario un inventario para obtener un permiso de aprovechamiento, pero los datos no se usaron en la toma de decisiones para el manejo forestal a largo plazo. Después de que se prohibiera el cambio de uso de la tierra en 1991, los sectores público y privado estuvieron de acuerdo en mejorar los procedimientos de manejo forestal mediante la inclusión de inventarios de existencias y reconocimientos topográficos como requisitos adicionales para la planificación del manejo, y la restricción del aprovechamiento a 60\% del volumen comercial sobre el diámetro mínimo de $60 \mathrm{~cm}$. Estas medidas se fijaron en 1992 y se apoyaron con incentivos gubernamentales en 1993 (Maginnis, Méndez y Davies, 1998).

En 1997 se fijó un ciclo de aprovechamiento mínimo de quince años y se cambiaron las pautas de regulación del rendimiento de una base por volumen a una base por árbol, con el fin de simplificarlas (el aprovechamiento se limita a 60\% del número de árboles comerciales por especie con valor comercial, por encima del diámetro mínimo de corta de 60 cm) (Maginnis et al., 1998).

El manejo forestal y la silvicultura, hoy en día, tratan de jugar un papel más relevante en la economía y en los medios de vida de las comunidades a partir del principio de la multifuncionalidad de los bosques y al asumir que la sostenibilidad del bosque considera los usos, creencias y costumbres de las personas, así como la necesidad de generar beneficios económicos y sociales a diferentes escalas que logren competir con los usos alternativos del suelo (Meza y Méndez, 2006).

\section{Aprovechamiento forestal}

El aprovechamiento forestal tiene como fin el uso racional de los bosques para extraer productos de forma sostenible, y trata de provocar el menor impacto posible; es por esto que el aprovechamiento de bosque se vislumbra como la herramienta más adecuada para el ordenamiento forestal (Hidalgo 2004).

Según Meza (2004), el aprovechamiento forestal es un sistema de producción, por lo tanto, tiene una serie de etapas bien definidas, las cuales deben interactuar entre sí en forma ordenada y lógica para lograr el objetivo señalado. Se le considera como el primer tratamiento silvicultural que se le aplica al bosque; este tratamiento, además de rendir ingresos, permite dinamizar el ecosistema mediante la apertura de claros. La calidad de la regeneración que se establezca en los claros dependerá de la planificación y cuidado con que se realice la primera intervención (Valerio, 1997).

La ejecución del aprovechamiento debe hacerse bajo una "estructura de servicio", de modo que las operaciones de cada etapa se realicen a fin de sacar el mejor provecho del producto proveniente de la etapa anterior y facilitar la labor siguiente. Para lograr esta estructura, es fundamental un proceso detallado de planificación de las actividades a ejecutar en cada etapa (Meza, 2004). El objetivo del aprovechamiento debe ser extraer la mayor cantidad de productos, de la mejor calidad, al menor costo y causando la mínima alteración al ambiente (Cordero, 1998).

Para realizar un aprovechamiento exitoso en bosques tropicales, es necesaria la planificación adecuada del método de extracción a utilizar. Según Castillo (|99|), el aprovechamiento de los bosques naturales se ve afectado por la variedad florística, el tamaño de los árboles, la densidad de la vegetación, el clima, la topografía de la zona, las vías de extracción y el acceso al área, así como la disponibilidad de mano de obra.

En el presente estudio se analizó la recuperación mostrada por el bosque diecinueve años después de aplicar cuatro sistemas de aprovechamiento, los cuales presentan variaciones en las operaciones de corta y extracción, que genera diferente grado de impacto en el bosque y en la masa arbórea remanente, como se presenta en el siguiente cuadro.

\section{Sistema de aprovechamiento con bueyes (SACB)}

El sistema de aprovechamiento con bueyes utiliza esta clase de animales en la extracción, los cuales son 
Cuadro I. Descripción de los tratamientos silviculturales empleados en los bosques de Mogos, Estero Guerra y Dos Brazos de Rincón (Cordero y Howard, 1990; Castillo 1991; Quesada y Castillo, 2010)

\begin{tabular}{|c|c|c|}
\hline Sistema de aprovechamiento & Simbología & Grado de perturbación al bosque \\
\hline Sistema de aprovechamiento con bueyes & SACB & - \\
\hline Sistema de aprovechamiento tradicional mejorado & SATM & -- \\
\hline Sistema de aprovechamiento tradicional con tractor & SATT & ---- \\
\hline Sistema de aprovechamiento tradicional con tractor & SABT & --- \\
\hline
\end{tabular}

*Donde: - = menor perturbación; - - = mediana perturbación; - - = alta perturbación; - - - - = muy alta perturbación.

equipados con cadenas y un arco para la extracción de árboles. Si es necesario, las trozas de longitudes mayores se cortan longitudinalmente por la mitad (Castillo y Cordero, 1990).

Debido a que las pistas de arrastre son angostas, generalmente lo único que se requiere es hacer la corta de la vegetación presente en la pista, sin necesidad de hacer movimiento de tierra, por lo que la alteración es mínima. Aun cuando la presión por unidad de superficie que ejercen sobre el suelo los animales sea mayor que la que ejerce una máquina, el área que necesitan para desplazarse es menor y el efecto negativo que causan es mínimo. Por la baja velocidad a la que viajan, se reducen los daños a los árboles remanentes (FAO, 1995).

\section{Sistema de aprovechamiento tradicional mejorado (SATM)}

El sistema de aprovechamiento tradicional mejorado es el más utilizado en la corta y arrastre en Costa Rica. Aunque las operaciones tradicionales son destructivas, al ser planificadas se pueden reducir los daños que se ocasionan al bosque. Por ello se debe procurar una óptima dirección de caída de los árboles, a fin de reducir los daños al sotobosque y a otros árboles del dosel superior, así como evitar que por una mala corta se quiebren o dañen las trozas. Para el arrastre de las trozas se hace uso del winch, el cual se utiliza para halar las trozas hacia las pistas de arrastre, con el propósito de reducir la presencia de la máquina en el bosque (Cordero, 1990).

\section{Sistema de aprovechamiento tradicional con tractor (SATT)}

El sistema de aprovechamiento tradicional con tractor es un método utilizado en Costa Rica, el cual es altamente destructivo y sin planificación, por lo que se ocasionan grandes daños al ecosistema (Castillo, 199|). Castillo (1996) menciona que en la ejecución del Proyecto Alternativas de Aprovechamiento de Bosque Natural. elaborado por el Instituto Tecnológico de Costa Rica en la Península de Osa en 1990, a pesar de que las labores de extracción no fueron planificadas, la selección de los árboles a cortar sí lo fue.

\section{Sistema de aprovechamiento con la combinación bueyes-tractor (SABT)}

El sistema de aprovechamiento con la combinación bueyes-tractor intenta reducir el área que perturba la máquina. En este caso, el tractor entra, construye las pistas de arrastre y extrae las trozas con diámetros mayores, mientras que los bueyes se encargan de extraer los diámetros menores a fin de reducir la presencia de la máquina en el bosque (Castillo, 1996).

\section{Crecimiento}

La determinación de las existencias en una masa forestal en un momento dado es de naturaleza estática, en la medida que no se considere la evolución de la masa a través del tiempo. Los resultados que se obtengan por sí solos atribuyen al bosque una naturaleza inerte que no corresponde a su condición de población viva o dinámica, y en permanente producción (Quesada, 200 I).

En bosques sometidos a manejo forestal, es importante determinar cuáles son las tasas de crecimiento que presentan las especies, con el objetivo de proyectar ciclos de corta y evaluar la aplicación de tratamientos silviculturales que estimulen el crecimiento de las especies (Quesada y Castillo, 20 I0). 
El crecimiento de los árboles se ve afectado por muchas variables, como pueden ser: competencia, suelo, edad, especie, genéticos, madurez, estado fitosanitario, entre otras (Quesada y Castillo, 20 I0). Sin embargo, en este informe no se detallarán, debido a que la variable que más afecta a la masa es la cosecha y las actividades que esta involucra.

\section{Mortalidad y reclutamiento}

El bosque húmedo y lluvioso tropical es un sistema dinámico. La polinización, la diseminación y la germinación forman parte de la experiencia diaria, al igual que la muerte y la caída de los grandes árboles del dosel (Guariguata y Kattan, 2002). Dentro de la dinámica del bosque se presenta la caída de un árbol o ramas, lo que produce una apertura en el dosel superior, ya sea de forma natural o por el aprovechamiento forestal, lo que permite el aumento de la penetración de luz en el piso del bosque y da origen, principalmente, al establecimiento de nueva regeneración y a la activación del crecimiento de los árboles que se encontraran suprimidos en el dosel. La regeneración puede reaccionar de dos maneras: unos podrán continuar su desarrollo, pero la gran mayoría no sobrevivirán debido a que no son las condiciones ideales para su desarrollo (Pérez, 2002).

La caída de un árbol no es un proceso totalmente aleatorio en el espacio. Los árboles que se encuentran más cercanos a la orilla de los claros tienen más probabilidades de caer que aquellos que se encuentran más alejados, además, tienden a caer hacia el centro del claro preexistente (Young y Hubbell, |99|). La continua formación de claros (de distintos tamaños) permitirá la coexistencia de un gran número de especies de árboles. La diferencia en la cantidad de luz disponible que se observa en función del tamaño del claro sugiere, además, que la formación de claros puede propiciar una gran diversidad de especies (Guariguata y Kattan, 2002).

El conocimiento de la mortalidad arbórea como mecanismo de funcionamiento de los ecosistemas boscosos es fundamental en la formación de modelos que permitan la generación de estrategias de manejo sostenible y su conservación. La mortalidad de los árboles ocurre en diferentes escalas de intensidad, espacio y tiempo, siendo el reflejo tanto de procesos endógenos (como la senescencia de los individuos), como de disturbios exógenos (rayos, tormentas y temporales) (Melo y Vargas, 2003).
Por otro lado, Melo y Vargas (2003) definen reclutamiento como la capacidad que tiene el bosque para incrementar el número de individuos y es una manifestación de la fecundidad de las especies, lo mismo que del crecimiento y sobrevivencia de los individuos juveniles. Desde el punto de vista silvicultural, el reclutamiento mide el número de individuos que anualmente sobrepasan el límite inferior de medición para una distribución de categorías de tamaño.

El reclutamiento, junto con la mortalidad, constituye uno de los aspectos más importantes de la dinámica de una población (Londoño y Jiménez 1999).

\section{Metodología}

\section{Descripción de los sitios de estudio}

La dinámica del crecimiento se realizó en tres bosque intervenidos ubicados en la Península de Osa, Costa Rica. Los sitios Estero Guerra y Los Mogos pertenecen al tercer distrito Sierpe, quinto cantón de Osa. El sitio Estero Guerra se encuentra en la zona de vida Bosque muy húmedo transición a basal, y el de Mogos en la zona de vida Bosque muy húmedo tropical (Vílchez y Rocha, 2004). El sitio Dos Brazos de Río Rincón, perteneciente al segundo distrito Puerto Jiménez, del sétimo cantón Golfito, se encuentra en la zona de vida Bosque muy húmedo premontano transición a basal. Los tres sitios son parte del Área de Conservación Osa (ACOSA) y se hallan dentro del Corredor Biológico Osa.

Para la evaluación de la dinámica, fueron establecidas parcelas permanentes de muestreo (PPM) en el año 1990 por Castillo ( $99 \mid$ ), como parte del "Proyecto Alternativas de Aprovechamiento Forestal" (Cordero y Howard, 1990). En esta ocasión se inventariaron todos los árboles con un diámetro mayor o igual a $30 \mathrm{~cm}$, medido a I,3 m de altura (d), y los individuos con $d \geq 10 \mathrm{~cm}$ en 0,1 ha de cada parcela donde se aplicó cada tratamiento. Esto se debe a que, inicialmente, la medición de los árboles tenía como objetivo su posterior aprovechamiento, realizado entre 1991 y 1992 en los tres sitios. La fracción de parcela, en la que se midió a partir de 10 $\mathrm{cm}$ de diámetro, tenía como fin posterior un estudio de la regeneración. 
Tipo de cobertura de las unidades de muestreo Estero Guerra, Dos Brazos de Rio Rincón y Los Mogos, Peninsula de Osa, Costa Rica, 2011.

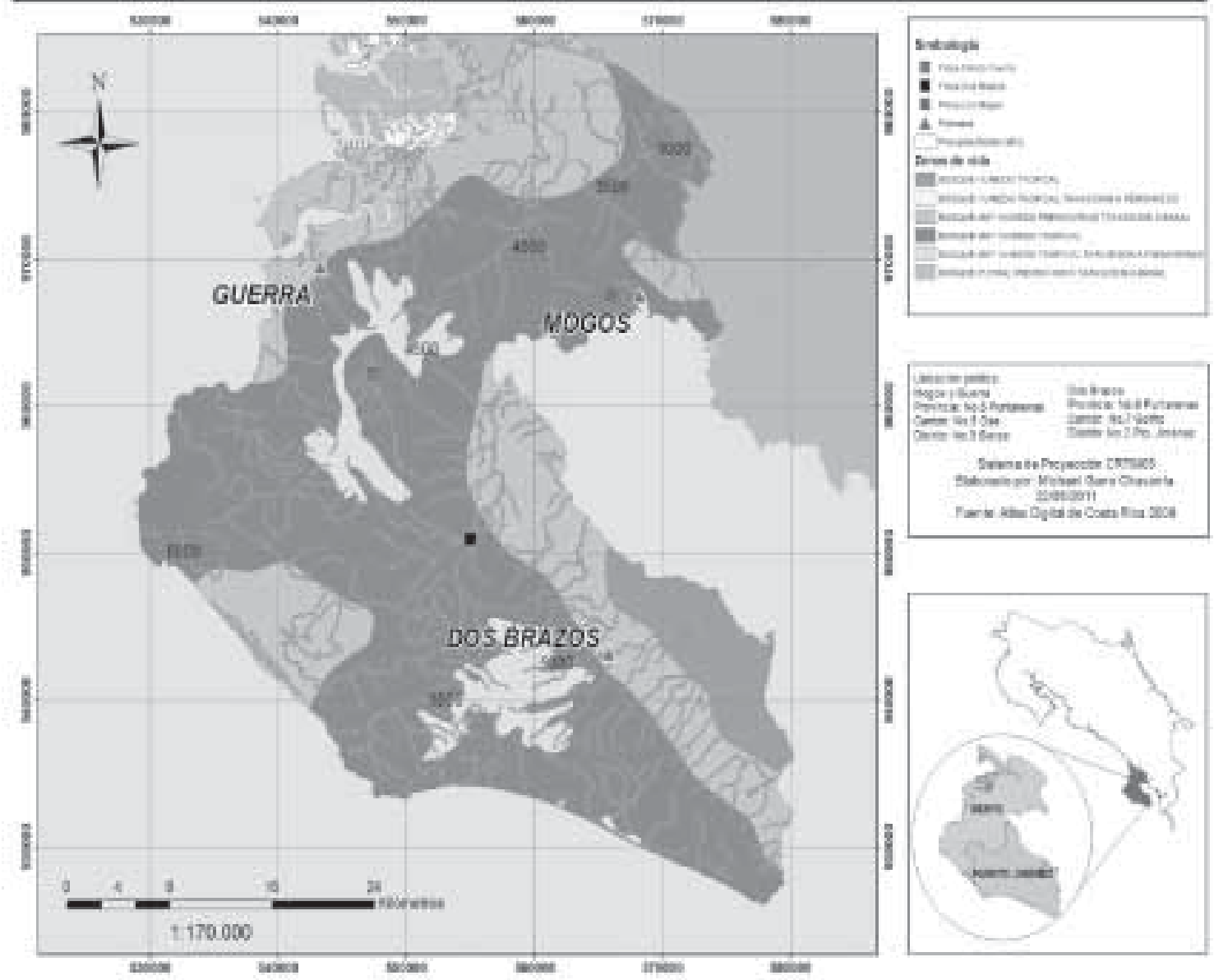

Figura I. Características ambientales de los sitios Estero Guerra, Dos Brazos de Río Rincón y Los Mogos, utilizados para el análisis de la dinámica del bosque húmedo tropical, Península de Osa, Costa Rica, 201 I.

La selección de los sitios realizada por Castillo (|99|) para establecer las parcelas se basó en características homogéneas en cuanto a topografía, composición florística inicial del bosque e hidrografía. Las PPM establecidas en Estero Guerra, Dos Brazos de Río Rincón y los Mogos se encuentran en las propiedades de Juan Marín Alpizar, José Ángel Mejías Sibaja y Carlos Montero Díaz.

Se establecieron cuatro PPM de una hectárea en cada sitio, rectangulares $(200 \times 50 \mathrm{~m})$ o cuadradas ( $100 \times 100$ m); dependiendo de la forma del terreno y facilidad para ubicarlas en cada sitio, cada una subdividida en 100 subparcelas de $10 \times 10 \mathrm{~m}$. Entre cada unidad experimental o parcela permanente se dejó como mínimo una zona de amortiguamiento de $25 \mathrm{~m}$. En cada PPM se realizaron cuatro sistemas de aprovechamientos, los cuales se detallan en el Cuadro 2.

La permanencia de las parcelas se ha logrado gracias al esfuerzo de los proyectos de investigación: Alternativas sistemas de aprovechamiento para bosques tropicales (Cordero y Howard, 1990); Establecimiento de parcelas permanentes de muestreo en bosque natural, para evaluar el aprovechamiento forestal, Península de Osa, Costa Rica. (Castillo, I99I); Comportamiento del bosque natural después del aprovechamiento forestal ejecutado entre 1992 y 1995 (Valerio, Salas y Castillo, 1995); Regulaciones al aprovechamiento forestal- REFORMA ejecutado entre 1995 y 1997 (Valerio et al, 1995); Caracterización de la vegetación del Parque Nacional Corcovado, Área de 
Cuadro 2. Sistemas de aprovechamiento forestal utilizados en cuatro parcelas permanentes de muestreo de una hectárea en los sitios Estero Guerra, Dos Brazos y Mogos, Península de Osa, Costa Rica, 201 I.

\begin{tabular}{|c|c|}
\hline Parcelas & Sistema de aprovechamiento forestal \\
\hline 1 & Con bueyes (SACB) \\
\hline 2 & Tradicional mejorado (SATM) \\
\hline 3 & Tradicional con tractor (SATT) \\
\hline 4 & Combinación Bueyes-tractor (SABT) \\
\hline
\end{tabular}

Conservación Osa, mediante un sistema de parcelas permanentes (Quesada y Castillo, 2004) ejecutado entre 1997 y 2000; Distribución y abundancia de árboles de dosel del bosque húmedo tropical en la Península de Osa, (Castillo et al, 2007); y, por último, el proyecto Demografía de especies comerciales de la Península de Osa ejecutado entre el 2005 y el 2008 (Quesada y Castillo, 20 I0).

\section{Crecimiento}

Para realizar el análisis del crecimiento de la masa arbórea después del aprovechamiento, se calculó el incremento corriente anual (ICA mm/ año) diamétrico en un periodo de cuatro años (2007-20 I I) con los individuos con $d \geq 10 \mathrm{~cm}$. Se analizaron los valores ICA promedio por sistema de aprovechamiento y en cada clase diamétrica.

\section{Mortalidad y reclutamiento (dinámica del bosque)}

En el análisis de los reclutamientos para cada uno de los sitios (Estero Guerra, Dos Brazos y Los Mogos), se utilizaron los datos de los individuos que alcanzaron los $10 \mathrm{~cm}$ de diámetro a la altura de 1,3 m en el 20II, y que no se encontraban en el 2007. Para el análisis de la mortalidad en cada uno de los sitios, se trabajó con los individuos presentes en el 2007 y que en las mediciones del 201 I no aparecieron.

Los resultados obtenidos de las tasas de mortalidad y reclutamiento se promediaron con el fin de obtener tasas anuales para cada sistema de aprovechamiento y, por medio de la diferencia entre estas dos tasas, se calculó el recambio anual para método de aprovechamiento.

El cálculo de la tasa de mortalidad anual se realizó por medio de la siguiente expresión:

$$
r_{m}=\left[1-\left(\frac{N_{S}}{N_{0}}\right)^{\frac{1}{t}}\right] x 100
$$

Donde

$r_{m}=$ Tasa anual de mortalidad expresada en porcentaje

$N_{0}=$ Número de individuos inicialmente inventariados

$N_{s}=$ Número de individuos inicialmente inventariados, sobrevivientes en un inventario posterior después de un intervalo $t$ de tiempo, $N_{s}=N_{0}-M_{u}$

$M_{u}=$ Número de individuos muertos durante el intervalo $t$ de tiempo

$t=$ Intervalo de tiempo en años, transcurrido entre los dos inventarios

El cálculo de la tasa de reclutamiento se realizó por medio de la siguiente expresión:

$$
r_{r}=\left[\left(\frac{N_{S}}{N_{0}}\right)^{\frac{1}{t}}-1\right] x 100
$$

Donde

$r_{r}=$ Tasa anual de reclutamiento expresada en porcentaje

$N_{0}=$ Número de individuos inicialmente inventariados

$N_{t}=$ Número de individuos inicialmente inventariados más los reclutados durante el periodo $t$ de tiempo, $N_{t}=N_{0}+1$ 
1 = Número de individuos reclutados durante el intervalo $t$ de tiempo

$t=$ Intervalo de tiempo en años, transcurrido entre los dos inventarios

\section{Análisis de los datos}

En el análisis estadístico de las variables descritas anteriormente se realizaron pruebas de normalidad Shapiro-Wilks modificadas para comprobar una distribución normal de cada conjunto de datos a evaluar y un análisis de varianza con los residuos absolutos de cada grupo de datos para comprobar homocedasticidad (homogeneidad en las varianzas). Una vez que se determinó que los datos presentaban una distribución normal y homocedasticidad, se realizó un análisis de varianzas para diseño en bloques completamente aleatorio y pruebas Tukey para cada variable, con el fin de determinar si existían diferencias significativas $(\alpha=0,05)$ entre los aprovechamientos en cada año. En las variables en que se compararon dos estados de bosque (1990 y 201 I), se realizaron pruebas $t$ apareadas para determinar si existían diferencias significativas $(\alpha=0,05)$ entre las variables de un año a otro

Cuadro 3. Incremento corriente anual (ICA) promedio en diámetro $(\mathrm{d} \geq 10 \mathrm{~cm})$ y desviaciones estándar en el periodo de quince a diecinueve años después de la cosecha bajo cuatro sistemas de aprovechamiento forestal en la Península de Osa, Costa Rica, 201 I

\begin{tabular}{|c|c|c|c|c|}
\hline \multirow{2}{*}{$\begin{array}{l}\text { Clases diamétricas } \\
\qquad(\mathrm{cm})\end{array}$} & \multicolumn{2}{|l|}{ SACB } & \multicolumn{2}{|l|}{ SATM } \\
\hline & ICA (mm/año) & $\mathrm{n}$ & ICA (mm/año) & $\mathrm{n}$ \\
\hline $10-20$ & $1,54 \pm 1,97$ & 859 & $1,64 \pm 2,33$ & 929 \\
\hline $20-30$ & $2,80 \pm 3,11$ & 274 & $3,30 \pm 3,54$ & 290 \\
\hline $30-40$ & $3,64 \pm 5,04$ & 124 & $4,11 \pm 4,42$ & 135 \\
\hline $40-50$ & $4,02 \pm 5,255$ & 74 & $5,41 \pm 6,79$ & 84 \\
\hline $50-60$ & $2,89 \pm 3,72$ & 56 & $3,39 \pm 4,32$ & 38 \\
\hline $60-70$ & $4,53 \pm 14,58$ & 32 & $3,51 \pm 3,55$ & 21 \\
\hline $70-80$ & $4,20 \pm 7,09$ & 24 & $2,44 \pm 4,43$ & 17 \\
\hline $80-90$ & $1,85 \pm 3,14$ & 5 & $3,15 \pm 5,95$ & 10 \\
\hline $90-100$ & $4,06 \pm 6,56$ & 4 & $2,68 \pm 5,52$ & 7 \\
\hline $100-110$ & & & 0 & 2 \\
\hline Promedio total & $3,28 \pm 5,61$ & 1452 & $2,96 \pm 4,53$ & 1533 \\
\hline \multirow{2}{*}{$\begin{array}{l}\text { Clases diamétricas } \\
\qquad(\mathrm{cm})\end{array}$} & \multicolumn{2}{|l|}{ SATT } & \multicolumn{2}{|l|}{ SABT } \\
\hline & ICA (mm/año) & $\mathrm{n}$ & ICA (mm/año) & $\mathrm{n}$ \\
\hline $10-20$ & $1,66 \pm 2,18$ & 823 & $1,89 \pm 2,40$ & 921 \\
\hline $20-30$ & $3,18 \pm 3,72$ & 261 & $3,6 \pm 3,82$ & 305 \\
\hline $30-40$ & $4,23 \pm 4,67$ & 131 & $4,62 \pm 5,71$ & 133 \\
\hline $40-50$ & $3,90 \pm 4,85$ & 57 & $4,4 I \pm 5,48$ & 70 \\
\hline $50-60$ & $4,4 I \pm 5,95$ & 51 & $3,89 \pm 5,37$ & 40 \\
\hline $60-70$ & $2,04 \pm 3,29$ & 33 & $4,94 \pm 6,73$ & 36 \\
\hline $70-80$ & $3,29 \pm 4,16$ & 20 & $4,85 \pm 5,23$ & 13 \\
\hline $80-90$ & $1,62 \pm 2,43$ & 6 & $4,37 \pm 5,45$ & 4 \\
\hline $90-100$ & $1,22 \pm 2,14$ & 8 & $2,75 \pm 3,89$ & 2 \\
\hline $100-110$ & $1,62 \pm 2,30$ & 2 & $3,87 \pm 1,24$ & 2 \\
\hline $110-120$ & 0 & 1 & 0 & 1 \\
\hline Promedio total & $2,47 \pm 3,57$ & 1393 & $3,56 \pm 4,53$ & 1527 \\
\hline
\end{tabular}

*SACB = Sistema de aprovechamiento con bueyes; SATM = Sistema tradicional mejorado, SATT = Sistema de aprovechamiento tradicional con tractor y SABT = Sistema de aprovechamiento bueyes-tractor. 
dentro de cada sistema de aprovechamiento. Todas las pruebas descritas se realizaron con el programa libre InfoStat, versión 20 I le.

\section{Crecimiento del bosque intervenido}

El crecimiento de las especies en el bosque está afectado por muchas variables, sin embargo, al evaluar bosques manejados, se incorpora la variable de la intervención y cómo esta provoca cambios en el comportamiento de la masa remanente, la cual se ve afectada por la capacidad de crecimiento de cada especie, donde la tolerancia tiene un rol importante (Castillo et al, 2007).

El Cuadro 3 presenta el crecimiento expresado en el promedio del incremento corriente anual (ICA) para un periodo de cuatro años, pasado un quinquenio desde la ejecución del aprovechamiento forestal.

El incremento encontrado para los bosques en los últimos cuatro años va desde los 2,47 a los 3,56 mm/ año, siendo el SATT el que presenta el menor valor y el SABT el mayor. Para los mismos tres sitios en un periodo de doce años ( $1995-2007)$, se reportan valores en el ICA diamétrico que varían de 2,82 a $3,21 \mathrm{~mm} /$ año (el SATM con el menor incremento y el SABT el valor más alto) (Quesada y Castillo, 2010).

Los datos reportados por estos autores y los presentados en el cuadro 6 muestran valores similares de ICA, tanto quince como diecinueve años después del aprovechamiento, sin embargo, se observa una disminución en el incremento diamétrico del sistema SATT y un aumento en el resto de los aprovechamientos (SACB, SATM y SABT); diecinueve años después del manejo, en ambos periodos de medición (1995-2007 y 200720II), el SABT es el que presenta el mayor ICA diamétrico.

El cuadro 4 muestra que no hay una clase diamétrica que presente valores máximos de ICA. Para todos los aprovechamientos en la clase diamétrica de 60$70 \mathrm{~cm}$, los sistemas SACB (4,53 mm/año) y SABT (4,94 mm/año) presentan picos de crecimiento en diámetro, mientras que los sistemas SATT (4,4l mm/año) y SATM $(5,41 \mathrm{~mm} / a n ̃ o)$ presentan los valores máximos en las clases $50-60 \mathrm{~cm}$ y $40-$ $50 \mathrm{~cm}$, respectivamente. Centeno (2010) reporta, para bosques después de dieciocho años de haber sido sometidos a aprovechamiento de bajo impacto, valores de ICA que van de 2,2 a 7,3 mm/año, presentando el mayor incremento en la clase de 70-80 para bosques en San Carlos, Costa Rica. Para bosques intervenidos bajo distintas intensidades en la Región de Boca Tapada, se reportan valores de ICA diamétrico de 3, I 2 a 3,8 mm/año en un periodo de ocho años (Quesada, 200I).

Este mismo autor indica que los máximos ICA se encontraban en las clases entre 40 y $70 \mathrm{~cm}$ de diámetro, mientras que en las clases siguientes se presentan valores menores a $1 \mathrm{~mm} / a n ̃ o$. Hernández y Castellanos (2006) encontraron valores en ICA

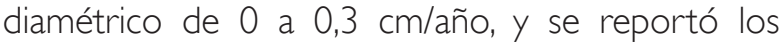
mayores valores en las clases de 30 a más de $50 \mathrm{~cm}$ de diámetro, para bosques primarios de la Guayana Venezolana. En relación con los datos mostrados

Cuadro 4. Comportamiento de la mortalidad y el reclutamiento (individuos por hectárea y tasa anual, en un periodo de cuatro años para tres bosques manejados bajo cuatro sistemas de aprovechamiento forestal en la Península de Osa, Costa Rica, 201 I.

\begin{tabular}{|c|c|c|c|c|c|}
\hline \multirow{2}{*}{ Aprov. } & \multicolumn{2}{|c|}{ Mortalidad } & \multicolumn{2}{|c|}{ Reclutamiento } & Tasa anual de \\
\cline { 2 - 5 } & $\mathbf{N}$ & Tasa (\%) & $\mathbf{N}$ & Tasa (\%) & 0,817 \\
\hline SACB & 19 & 0,938 & 38 & 1,755 & 1,090 \\
\hline SATM & 26 & 1,163 & 52 & 2,252 & 0,428 \\
\hline SATT & 31 & 1,491 & 42 & 1,919 & $-0,060$ \\
\hline SABT & 36 & 1,611 & 36 & 1,551 & 0,569 \\
\hline Promedio & 28 & 1,301 & 42 & 1,869 & (\%) \\
\hline
\end{tabular}

*SACB = Sistema de aprovechamiento con bueyes, SATM = Sistema tradicional mejorado, SATT = sistema de aprovechamiento tradicional con tractor y SABT = sistema de aprovechamiento bueyes-tractor. 
por estos autores, el crecimiento de los bosques en estudio es mayor en los diferentes sistemas de aprovechamiento durante los últimos cuatro años.

El análisis estadístico demostró que el valor ICA presenta una distribución normal y homocedasticidad; por otro lado, el análisis de varianza indica que no existen diferencias significativas entre los ICA de cada sistema de aprovechamiento $(p=0,3410)$.

\section{Mortalidad y reclutamiento}

El análisis de la mortalidad y el reclutamiento se realizó con los datos entre los quince años (2007) y diecinueve años (20 I I) después del aprovechamiento; se contó con un total de 335 muertos y 504 ingresos para los tres sitios y para los cuatro sistemas de aprovechamiento, Las tasas anuales de mortalidad, reclutamiento y recambio para cada tipo de manejo se presentan en el cuadro 4.

EI SABT presenta la mayor tasa de mortalidad y la menor tasa de reclutamiento, aún menor que el porcentaje anual de mortalidad para el mismo periodo de tiempo, lo que da como resultado una tasa de recambio negativa. Por otro lado, el SATM muestra la mayor tasa anual de recambio con una tasa anual de reclutamiento de casi el doble de la mortalidad.

En un bosque en su estado clímax, el volumen y la composición florística se mantienen más o menos constantes, y ocurre solamente una sustitución continua de unos individuos, generalmente los más viejos por otros (Castillo et al 2007). Lamprecht (1990) establece que, en los bosques maduros, los incrementos y la aparición de nuevos individuos tienden solo a sustituir al material muerto y no necesariamente aumentan en el volumen; en otras palabras, la tasa de recambio (por ejemplo, el área basal) es prácticamente cero.

Según esto, el SACB y el SATT son los que presentan valores más cercanos a un bosque primario. En la Estación Biológica Sirena se reportan tasas de mortalidad entre $4,21 \%$ y $2,48 \%$, y de reclutamiento entre $4,46 \%$ y $2,35 \%$ para bosques primarios ( $d=5$ $\mathrm{cm}$ ), lo que revela valores muy similares de ingresos y mortalidad (Quesada y Castillo, 2004).

La tasa de recambio anual media para los tres sitios tiene un valor de 0,569\%, por lo que la tendencia es que el reclutamiento es mayor a la mortalidad; este valor de recambio cercano a 0 muestra un bosque menos dinámico, y que el porcentaje de ingresos y mortalidad está cercano a equilibrarse, comportamiento esperado para un bosque primario; también indica que no se han presentado grandes disturbios de origen natural ni humano en los últimos años.

Pérez (2002), para la región de Mogos, muestra tasas anuales de mortalidad de 0,76\% a 2,25\%; porcentajes de reclutamiento que van de 2,32 a 3,83 y una tasa anual de recambio media de 1,66\% para las mismas unidades de muestreo del presente estudio, diez años después de ejecutados los aprovechamientos. Para bosques intervenidos (diez años) bajo distintas intensidades en la región Huetar Norte de Costa Rica, Quesada (200I) reporta tasas de recambio que van de $-0,8 \%$ a $10,2 \%$.

Los resultados expuestos por estos autores muestran un bosque más dinámico diez años después del aprovechamiento forestal, donde la diferencia entre las tasas anuales de mortalidad e ingresos recambio son mayores que las mostradas en el cuadro 4.

Para bosques intervenidos de 18 años de Pital de San Carlos, sometidos a un tratamiento silvicultural (I año después del aprovechamiento) "refinamiento", se reportan tasas anuales de mortalidad y reclutamiento de $2,42 \%$ y 2,09\% respectivamente, en un periodo de 8 años (Centeno, 20l0).

La distribución diamétrica para la mortalidad por hectárea muestra que el sistema SABT presenta los mayores valores para las dos primeras clases diamétricas, seguido por los aprovechamientos SATT, SATM y SACB. A partir de los $30 \mathrm{~cm}$ de diámetro, la mortalidad disminuye de forma más marcada y la cantidad de individuos por hectárea varía en cada aprovechamiento conforme aumenta el diámetro.

En la clase de 50-60 cm, el SATT no presenta muerte de individuos en una hectárea, al igual que el SABT pero con los individuos entre 60 y 70 $\mathrm{cm}$ de diámetro; mientras que con el SACB no se presenta mortalidad por hectárea para la última clase diamétrica; por lo tanto, el SATM es el único aprovechamiento que presenta mortalidad en todas las clases diamétricas.

El SABT es el que presenta la mortalidad más alta en todas las clases diamétricas, contrario al SACB y al SATM, los cuales producen un impacto menor, lo que podría estar relacionado con una mayor mortalidad en las especies pioneras de un ciclo de vida corto, 
que regeneraron en mayor cantidad poco después del aprovechamiento, el cual había producido más perturbación y formado más claros (SABT).

\section{Conclusiones}

El incremento para los bosques en estudio es de 2,47 a los 3,56 mm/año, y no se encontraron diferencias significativas entre los ICA de cada sistema de aprovechamiento.

La tasa de recambio anual media para los tres sitios tiene un valor de 0,569\%.

\section{Bibliografía}

Castillo, M. (199|) Establecimiento de Parcelas Permanentes de Muestreo en Bosque Natural. Para Evaluar el Aprovechamiento Forestal, Península de Osa Costa Rica. Cartago: Instituto Tecnológico de Costa Rica. p I49.

Castillo, M. (1996) Comportamiento del Bosque Natural Después del Aprovechamiento Forestal en Tres Sitios de la Península de Osa Costa Rica. Tesis de licenciatura Instituto Tecnológico de Costa Rica, Cartago. p 76.

Castillo, M. (2003). Recuperación del Área Basal Afectada por el Aprovechamiento de un Bosque Natural, Península de Osa, Costa Rica. I Congreso Silvicultural. Manejo Silvicultural: Para la Conservación de los Bosques. Recuperado de http://www.una.ac.cr/inis/silvi. htm.

Castillo, M., Fallas, A. \& Quesada, R. (2007). Distribución y abundancia de árboles de dosel del bosque húmedo tropical en la Península de Osa. Informe Final. Instituto Tecnológico de Costa Rica, Instituto Nacional de Biodiversidad-CTCBO, Critical Ecosystem Partnership Fund. Cartago. Costa Rica. p 124.

Centeno, F. (2010). Evaluación de la Aplicación de Tratamientos Silviculturales en Bosques Naturales de la Región Huetar Norte de Costa Rica. Informe de Práctica de Especialidad, Escuela de Ingeniería Forestal, Instituto Tecnológico de Costa Rica, Cartago, Costa Rica, p 84.

Cordero, W. \& Howard, A. (1990). Alternativas sistemas de aprovechamiento para bosques tropicales. Propuesta de Proyecto de Investigación. Departamento de Ingeniería Forestal. p 10.

Cordero, W. (1998). Aprovechamiento forestal. Instituto Tecnológico de Costa Rica. Serie de Apoyo Académico. p.I0I.
Food and Agriculture Organization of the United Nations (FAO). (1995). Uso de bueyes en operaciones de aprovechamiento forestal en áreas rurales de Costa Rica. Depósito de documentos de la FAO, V4925. Obtenido desde: http://www.fao.org/docrep/v4925S/ v4925S00.htm.

Guariguata, M. \& Kattan, G. (2002). Ecología y Conservación de Bosques Neotropicales. (p 69l). Cartago: LUR..

Hernandez, L \& Castellanos, H. (2006). Crecimiento diamétrico arbóreo en bosques de Sierra de Lema, Guayana Venezolana: Primeras evaluaciones. Interciencia. 3 I ( I I): 279 - 289.

Hidalgo, F. (2004). Caracterización y potencial de utilización de árboles caídos en los bosques de la Reserva Forestal de Golfo Dulce, Península de O s a , Puntarenas, Costa Rica. Informe de Práctica de Especialidad, Escuela de Ingeniería Forestal, Instituto Tecnológico de Costa Rica, Cartago, Costa Rica, 2004.

Lamprecht, H. (1990). Silvicultura en los Trópicos: Los Ecosistemas Forestales en los Bosques Tropicales y sus especies arbóreas -posibilidades y métodos para un aprovechamiento sostenido. (Antonio Carrillo, Trad.) ( $\mathrm{p}$ 335) Eschborn: Deutsche Gesellschaft für Technische Zusammenarbeit.

Londoño, A.C. \& Jiménez, E.M. (1999). Efecto del tiempo entre los censos sobre la estimación de las tasas anuales de mortalidad y de reclutamiento de árboles (periodos de I, 4 y 5 años). Crónica Forestal y del Medio Ambiente. No I4. p 4I-58.

Louman, B., Quirós, D. \& Nilsson, M. (200 I). Silvicultura de bosques latifoliados tropicales con énfasis en América Central. (p 265) Turrialba: CATIE.

Maginnis, S., Méndez, J. \& Davies, J. (1998). Manual para el manejo de bloques pequeños de bosque húmedo tropical (con especial referencia a la Zona Norte de Costa Rica). (p 208). San Carlos: CODEFORSA.

Melo, O \& Vargas, R. (2003). Evaluación ecológica y silvicultural de ecosistemas boscosos. Ibagué, CO, Universidad de Tolima. I 83 p.

Meza, A. (2004). El aprovechamiento de plantaciones forestales: un sistema de producción. Kurú: Revista Forestal. I (3):I. Recuperado de: http://www.tec.cr/ sitios/Docencia/forestal/Revista_Kuru/anteriores/ anterior3/pdf/Solucion\%202.pdf.

Meza, V., Méndez, J. (2004). Segundas cosechas bajo un sistema policiclico de manejo para bosques húmedos tropicales. Región Huetar Norte, Costa Rica. (en línea). Kurú: Revista Forestal. 3(9): I . Consultado 10 de julio 20II. Obtenido desde: http://www.tec.cr/sitios/Docencia/forestal/Revista_ Kuru/anteriores/anterio r9/index.htm 
Pérez, R. (2002). Evaluación del Impacto del Aprovechamiento Forestal en Los Mogos, Península de Osa, Costa Rica. Informe final de proyecto. Instituto Tecnológico de Costa Rica. Cartago, Costa Rica. p 96.

Quesada, R. (1997). Struktur and Dynamic eines Tropischen Feuchtwaldes nach Holznutzung in Costa Rica. Tesis de Doctorado, Universidad George-August, Göttingen, Deutschland. Cuvillier Verlang Göttingen, p 137.

Quesada, M. (2001). Dinámica del bosque muy húmedo tropical diez años después de la intervención forestal en la Región Huetar Norte de Costa Rica. Informe Final proyecto de Investigación. Escuela de Ingeniería Forestal, Vicerrectoría de Investigación y Extensión, Instituto Tecnológico de Costa Rica. Cartago, Costa Rica. $p$ I 27.

Quesada, R. \& Castillo, M. (2004). Caracterización de la vegetación del Parque Nacional Corcovado, Área de Conservación Osa, mediante un sistema de parcelas permanentes de muestreo. Informe Final proyecto de Investigación, Escuela de Ingeniería Forestal, Vicerrectoría de Investigación y Extensión, Instituto Tecnológico de Costa Rica. Cartago, Costa Rica. p 7I.
Quesada, R. \& Castillo, M. (2010). Demografía de especies maderables de la Península de Osa. Informe final. Instituto Tecnológico de Costa Rica. Cartago, Costa Rica. p | 32.

Valerio, J., Salas, C. \& Castillo, M. (1995). Comportamiento del bosque natural después del aprovechamiento forestal. Informe final. Instituto Tecnológico de Costa Rica, Centro de Investigación en Integración Bosque Industria. Cartago, Costa Rica. p I 10.

Valerio, J. \& Salas, C. (1998). Selección de prácticas silviculturales para bosques tropicales: Manual técnico (Segunda edición corregida y aumentada), Santa Cruz: Proyecto BOLFOR. p 77.

Vílchez, B. \& Rocha, O. (2004). Fenología y biología reproductiva del nazareno (Peltogyne purpurea Pittier) en un bosque intervenido de la Península de Osa, Costa Rica, América Central. Kurú: Revista Forestal. I(I):I-10. Recuperado de http://www.tec.cr/sitios/ Docencia/forestal/Revista_Kuru/ anteriores/anterior l/ pdf/MILCHEZ6\%20feb.pdf

Young, T. \& Hubbell, S. (|99|). Crown asymmetry, tree falls and repeat disturbance of broad-leaved forest gaps. Ecology, 72: | 464- | 47|. 